\title{
Numerical Modeling of an Influenza Epidemic Model with Vaccination and Diffusion
}

\author{
Dussadee Somjaivang and Settapat Chinviriyasit
}

\begin{abstract}
The SVEIR (susceptible vaccinated exposed infectious recovered) influenza epidemic model involving non-linear ordinary differential equations is studied and extended to incorporate diffusion in one-space dimension to enable the geographic spread of the disease in a population first-order in time and second-order in spaces based on finite difference method are constructed to obtained the numerical solution of the proposal model. A parallel implementation procedure is studied and the proposed model is simulated by using the constructed method in order to investigate the rate of vaccination.
\end{abstract}

Index Terms-SVEIR epidemic model, finite difference method, parallel implementation.

\section{INTRODUCTION}

The study of disease occurrence is called epidemiology. An epidemic is an unusually large, short-term outbreak of a disease. A disease is called endemic if it persists in a population. Over the last fifty years, epidemic models have been received a great attention in mathematical ecology. It is well-known that the involvement of many scientists in the development of a wide range of better models to describe the evolution of various types of epidemics [1]-[5].The propagation of an infection governed by these models do not incorporate any structure due to age, sex, variability of infectivity, or spatial position.

Several extensions of the model have been consider, focusing on better describing many specific features of the disease kinetics, though few have focused on spatial issues. Dietz and Schenzle [6] have pointed out that realistic epidemic models should consider vaccination programmes. There are many epidemic models which consider the effect of vaccination in epidemic models. Usually most of the models assume vaccination of individuals of all ages a constant rate or vaccination of specified fractions of individuals at given ages [7]-[10]. They have focused on the identification of what proportion of the population would need to be vaccinated to prevent influenza epidemics and pandemics. Anderson and May [11], [12] have shown the impact of different immunization policies on the age-specific incidence of rubella and measles. Greenhalgh and Das [13] studied SIR model with susceptibles divided into two classes. Samsuzzoha et al. [14] proposed the influenza model with

Manuscript received November 9, 2013; revised January 15, 2014. This work was supported in part by the National Centre of Exellence in Mathematics under the Commission on Higher Educational, Ministry of Education, Thailand

The authors are with the Department of Mathematics at King Mongkut's University of Technology Thonburi, Bangkok, 10140 Thailand (e-mail: dsd_sjw@hotmail.co.th, settapat.chi@kmutt.ac.th). vaccination and analyzed a model structured by spatial position in a bounded one-dimensional environment. These studies, however, gave no detail on the numerical method used to solve the resulting nonlinear initial-boundary value problems.

The Influenza epidemic model with vaccination and diffusion by Samsuzzoha et al. [14],

$$
\begin{aligned}
& \frac{\partial S}{\partial t}=-\beta_{1} E S-\beta_{2} I S+\alpha I S-\phi S-r S+\delta R+\theta V+r+d_{1} \frac{\partial^{2} S}{\partial x^{2}} \\
& \frac{\partial V}{\partial t}=-\beta_{3} E V-\beta_{4} I V+\alpha I V-r V-\theta V+\phi S+d_{2} \frac{\partial^{2} V}{\partial x^{2}} \\
& \frac{\partial E}{\partial t}=\beta_{1} E S+\beta_{2} I S+\beta_{3} E V+\beta_{4} I V+\alpha I E-w_{1} E+d_{3} \frac{\partial^{2} E}{\partial x^{2}} \\
& \frac{\partial I}{\partial t}=\sigma E-w_{2} I+\alpha I^{2}+d_{4} \frac{\partial^{2} I}{\partial x^{2}} \\
& \frac{\partial R}{\partial t}=\kappa E+\gamma I-r R-\delta R+\alpha I R+d_{5} \frac{\partial^{2} R}{\partial x^{2}}
\end{aligned}
$$

where $\beta_{1}=\beta \beta_{E}, \beta_{2}=\beta \beta_{I}, \beta_{3}=\beta \beta_{E} \beta_{V}, \beta_{4}=\beta \beta_{I} \beta_{V}$, $w_{1}=r+\kappa+\sigma$ and $w_{2}=r+\alpha+\gamma$.

The initial conditions are of the form,

$$
\begin{gathered}
S(x, 0)=S_{0}(x), V(x, 0)=V_{0}(x), E(x, 0)=E_{0}(x), \\
I(x, 0)=I_{0}(x), R(x, 0)=R_{0}(x),
\end{gathered}
$$

for $-L \leq x \leq L$,

and the boundary conditions are

$$
\frac{\partial S( \pm 2, t)}{\partial x}=\frac{\partial V( \pm 2, t)}{\partial x}=\frac{\partial E( \pm 2, t)}{\partial x}=\frac{\partial I( \pm 2, t)}{\partial x}=\frac{\partial R( \pm 2, t)}{\partial x}=0, t>0 .
$$

TABLE I: MODEL PARAMETERS [9]

\begin{tabular}{lll}
\hline \hline Parameters & Description & Value \\
\hline$\beta$ & Contact rate & 0.514 \\
$\beta_{E}$ & $\begin{array}{l}\text { Ability to cause infection by exposed } \\
\text { individuals }\end{array}$ & 0.250 \\
$\beta_{I}$ & Ability to cause infection by infectious & 1.000 \\
$\beta_{V}$ & individuals & \\
$\sigma$ & Ability to cause infection by vaccination & 0.1 \\
$\gamma$ & individuals & 0.500 \\
$\delta$ & Rate of latency & 0.200 \\
$\mu$ & Rate of clinically ill & $1 / 365$ \\
$r$ & Rate of duration of immunity loss & $5.5 \times 10^{-8}$ \\
$\kappa$ & Natural mortality rate & $7.140 \times 10^{-5}$ \\
$\alpha$ & Birth rate & $1.857 \times 10^{-4}$ \\
$\theta$ & Recovery rate of latents & $9.3 \times 10^{-6}$ \\
$\phi$ & Flu induced mortality rate & $1 / 365$ \\
\hline \hline
\end{tabular}




\section{NUMERICAL METHODS}

\section{A. Discretization and Notations}

A solution of the system of partial equation (1)-(3) may be computed by finite-difference methods by discretizing the space interval $[-L, L]$ into $M$ sub-intervals each of width $h>0$, and the time interval $t \geq 0$ is discretized in steps each of length $\ell>0$.

The open rectangle

$$
\Omega=[-L \leq x \leq L] \times[t>0]
$$

and its boundary $\partial \Omega$, consisting of the lines $\mathrm{x}=-L, x=L$ and

$t=0$, are covered by a rectangular grid having coordinates of the form $(x, t)=\left(x_{m}, t_{n}\right)=(-L+m h, n l)$

where

$x_{m}=-L+m h(m=0,1,2, \ldots, M)$ and $t_{n}=n \ell(n=0,1,2,3, \ldots)$.

The solutions of (1)-(3) at the typical mesh point $\left(x_{m}, t_{n}\right)$ are

$$
S\left(x_{m}, t_{n}\right), V\left(x_{m}, t_{n}\right), E\left(x_{m}, t_{n}\right), I\left(x_{m}, t_{n}\right), R\left(x_{m}, t_{n}\right)
$$

denoted by

$$
S_{m}^{n}, V_{m}^{n}, E_{m}^{n}, I_{m}^{n}
$$

and $\tilde{R}_{m}^{n}$ respectively. The solutions of numerical approximations at the same mesh point will be denoted by

$$
\widetilde{R}_{m}^{n}, \widetilde{V}_{m}^{n}, \widetilde{E}_{m}^{n}, \widetilde{I}_{m}^{n}
$$

and $R_{m}^{n}$ respectively.

\section{B. Construction of Method}

Finite-difference methods are constructed by approximating all time derivative in (1) by the first-order forward-difference replacement

$$
\frac{\partial u}{\partial t}=\frac{u(x, t+\ell)-u(x, t)}{\ell}+O(\ell) \quad \text { as } \ell \rightarrow 0,
$$

and the space derivative by the second-order approximant

$$
\frac{\partial^{2} u}{\partial x^{2}}=\frac{u(x-h, t+\ell)-2 u(x, t+\ell)+u(x+h, t+\ell)}{h^{2}}+O\left(h^{2}\right)
$$

as $h \rightarrow 0$

in which

$$
u(x, t)=S(x, t) \text { or } V(x, t) \text { or } E(x, t) \text { or } I(x, t) \text { or } R(x, t)
$$

Using (4) and (5) and making appropriate approximations for the right hand-side functions of the model (1), we obtain

$$
\begin{gathered}
\frac{\tilde{S}_{m}^{n+1}-\tilde{S}_{m}^{n}}{\ell}=\left(-\beta_{1} \tilde{E}_{m}^{n}-\beta_{2} \tilde{I}_{m}^{n}+\alpha \tilde{I}_{m}^{n}-\phi-r\right) \tilde{S}_{m}^{n}+\delta \tilde{R}_{m}^{n}+\theta \tilde{V}_{m}^{n}+r \\
\quad+d_{1}\left[\frac{\tilde{S}_{m-1}^{n+1}-2 \tilde{S}_{m}^{n+1}+\tilde{S}_{m+1}^{n+1}}{h^{2}}\right]
\end{gathered}
$$

$$
\begin{aligned}
\frac{\tilde{V}_{m}^{n+1}-\tilde{V}_{m}^{n}}{\ell} & =\left(-\beta_{2} \beta \tilde{E}_{m}^{n}-\beta_{3} \tilde{I}_{m}^{n}+\alpha \tilde{I}_{m}^{n}-r-\theta\right) \tilde{V}_{m}^{n}+\phi \tilde{S}_{m}^{n} \\
& +d_{2}\left[\frac{\tilde{V}_{m-1}^{n+1}-2 \tilde{V}_{m}^{n+1}+v_{m+1}^{n+1}}{h^{2}}\right]
\end{aligned}
$$

$\frac{\tilde{E}_{m}^{n+1}-\tilde{E}_{m}^{n}}{\ell}=\left(\beta_{1} \tilde{S}_{m}^{n}+\beta_{3} \tilde{V}_{m}^{n}+\alpha \tilde{I}_{m}^{n}-w_{1}\right) \tilde{E}_{m}^{n}+\beta_{2} \tilde{I}_{m}^{n} \tilde{S}_{m}^{n}+\beta_{4} \tilde{I}_{m}^{n} \tilde{V}_{m}^{n}$

$$
+d_{3}\left[\frac{\tilde{E}_{m-1}^{n+1}-2 \tilde{E}_{m}^{n+1}+\tilde{E}_{m+1}^{n+1}}{h^{2}}\right]
$$

$$
\frac{\tilde{I}_{m}^{n+1}-\tilde{I}_{m}^{n}}{\ell}=\left(-w_{2}+\alpha \tilde{I}_{m}^{n}\right) \tilde{I}_{m}^{n}+\sigma \tilde{E}_{m}^{n}
$$

$$
+d_{4}\left[\frac{\tilde{I}_{m-1}^{n+1}-2 \tilde{I}_{m}^{n+1}+\tilde{I}_{m+1}^{n+1}}{h^{2}}\right]
$$

$$
\frac{\tilde{R}_{m}^{n+1}-\tilde{R}_{m}^{n}}{\ell}=\left(-r-\delta+\alpha \tilde{I}_{m}^{n}\right) \tilde{R}_{m}^{n}+\kappa \tilde{E}_{m}^{n}+\gamma \tilde{I}_{m}^{n}
$$

$$
+d_{5}\left[\frac{\tilde{R}_{m-1}^{n+1}-2 \tilde{R}_{m}^{n+1}+\tilde{R}_{m+1}^{n+1}}{h^{2}}\right]
$$

which, after rearranging, becomes

$$
\begin{aligned}
& -d_{1} p \tilde{S}_{m-1}^{n+1}+\left(1+2 d_{1} p\right) \tilde{S}_{m}^{n+1}-d_{1} p \tilde{S}_{m-1}^{n+1} \\
& =\left[1-\left(\tilde{E}_{m}^{n}+\beta_{2} \tilde{I}_{m}^{n}+\alpha \tilde{I}_{m}^{n}-\phi-r\right) \ell\right] \tilde{S}_{m}^{n} \\
& +r \ell+\delta \ell \tilde{R}_{m}^{n}+\theta \ell \tilde{V}_{m}^{n}
\end{aligned}
$$

$$
\begin{aligned}
& -d_{2} p \tilde{V}_{m-1}^{n+1}+\left(1+2 d_{2} p\right) \tilde{V}_{m}^{n+1}-d_{2} p v_{m-1}^{n+1} \\
& =\left[1+\left(-\beta_{2} \beta \tilde{E}_{m}^{n}-\beta_{3} \tilde{I}_{m}^{n}+\alpha \tilde{I}_{m}^{n}-r-\theta\right) \ell\right] \tilde{V}_{m}^{n} \\
& +\phi \ell \tilde{S}_{m}^{n}
\end{aligned}
$$

$$
\begin{aligned}
& -d_{3} p \tilde{E}_{m-1}^{n+1}+\left(1+2 d_{3} p\right) \tilde{E}_{m}^{n+1}-d_{3} p \tilde{E}_{m-1}^{n+1} \\
& =\left[1+\left(\beta_{1} \tilde{S}_{m}^{n}+\beta_{3} \tilde{V}_{m}^{n}+\alpha \tilde{I}_{m}^{n}-w_{1}\right) \ell\right] \tilde{E}_{m}^{n} \\
& +\beta_{2} \ell \tilde{I}_{m}^{n} \tilde{S}_{m}^{n}+\beta_{4} \ell \tilde{I}_{m}^{n} \tilde{V}_{m}^{n} \\
& -d_{4} p \tilde{I}_{m-1}^{n+1}+\left(1+2 d_{4} p\right) \tilde{I}_{m}^{n+1}-d_{4} p \tilde{I}_{m-1}^{n+1} \\
& =\left[1+\left(-w_{2}+\alpha \tilde{I}_{m}^{n}\right) \ell\right] \tilde{I}_{m}^{n}+\sigma \ell \tilde{E}_{m}^{n}
\end{aligned}
$$




$$
\begin{aligned}
& -d_{5} p \tilde{R}_{m-1}^{n+1}+\left(1+2 d_{5} p\right) \tilde{R}_{m}^{n+1}-d_{5} p \tilde{R}_{m-1}^{n+1} \\
& =\left[1+\left(-r-\delta+\alpha \tilde{I}_{m}^{n}\right) \ell\right] \tilde{R}_{m}^{n}+\kappa \ell \tilde{E}_{m}^{n}+\gamma \ell \tilde{I}_{m}^{n}
\end{aligned}
$$

where $p=\ell / h^{2}$ and $\mathrm{m}=0,1,2, \ldots, M ; n=0,1,2, .$.

\section{The Local Truncation Error}

$$
\begin{aligned}
& \mathscr{L}_{S}[S(x, t), V(x, t), E(x, t), I(x, t), R(x, t) ; h, \ell] \\
& \mathscr{L}_{V}[S(x, t), V(x, t), E(x, t), I(x, t), R(x, t) ; h, \ell] \\
& \mathscr{L}_{E}[S(x, t), V(x, t), E(x, t), I(x, t), R(x, t) ; h, \ell] \\
& \mathscr{L}_{I}[S(x, t), V(x, t), E(x, t), I(x, t), R(x, t) ; h, \ell] \\
& \mathscr{L}_{R}[S(x, t), V(x, t), E(x, t), I(x, t), R(x, t) ; h, \ell]
\end{aligned}
$$

associated with (11)-(14) at the point $(x, t)=\left(x_{m}, t_{n}\right)$, which may be written down from (6)-(10) are given by

$$
\begin{aligned}
\mathscr{L}_{S} & =\frac{S(x, t+\ell)-S(x, t)}{\ell} \\
& -r+\left(\beta_{1} E(x, t)+\beta_{2} I(x, t)+\alpha I(x, t)-\phi-r\right) S(x, t)-\delta R(x, t) \\
& -\theta V(x, t)-d_{1}\left[\frac{S(x-h, t+\ell)-2 S(x, t+\ell)+S(x+h, t+\ell)}{h^{2}}\right] \\
& -S_{t}+r-\left(\beta_{1} E(x, t)+\beta_{2} I(x, t)+\alpha I(x, t)-\phi-r\right) S(x, t) \\
& +\delta R(x, t)+\theta V(x, t)+d_{1} S_{x x}
\end{aligned}
$$

$$
\begin{aligned}
\mathscr{L}_{V}= & \frac{V(x, t+\ell)-V(x, t)}{\ell} \\
& -\left(-\beta_{2} \beta E(x, t)-\beta_{3} I(x, t)+\alpha I(x, t)-r-\theta\right) V(x, t)-\phi S(x, t) \\
& -d_{2}\left[\frac{V(x-h, t+\ell)-2 V(x, t+\ell)+V(x+h, t+\ell)}{h^{2}}\right] \\
& -V_{t}-\beta_{2} \beta E(x, t) V(x, t)-\beta_{3} I(x, t) V(x, t)+\alpha I(x, t) V(x, t) \\
& -r V(x, t)-\theta V(x, t)+\phi S(x, t)+d_{2} V_{x x}
\end{aligned}
$$

$$
\mathscr{L}_{E}=\frac{E(x, t+\ell)-E(x, t)}{\ell}
$$

$-\left(\beta_{1} E(x, t)+\beta_{3} V(x, t)+\alpha I(x, t)-w_{1}\right) E(x, t)-\beta_{2} I(x, t) S(x, t)$

$-\beta_{4} I(x, t) V(x, t)-d_{3}\left[\frac{E(x-h, t+\ell)-2 E(x, t+\ell)+E(x+h, t+\ell)}{h^{2}}\right]$

$-E_{t}+\beta_{1} E(x, t) S(x, t)+\beta_{2} I(x, t) S(x, t)+\beta_{3} E(x, t) V(x, t)$

$+\beta_{4} I(x, t) V(x, t)+\alpha I(x, t) E(x, t)-w_{1} E(x, t)+d_{3} E_{x x}$

$$
\begin{aligned}
\mathscr{L}_{I}=\frac{I(x, t+\ell)-I(x, t)}{\ell} & \\
& -\left(-w_{2}+\alpha I(x, t) I(x, t)-\sigma E(x, t)\right. \\
& -d_{4}\left[\frac{I(x-h, t+\ell)-2 I(x, t+\ell)+I(x+h, t+\ell)}{h^{2}}\right] \\
& -I_{t}+\sigma E(x, t)-w_{2} I(x, t)+\alpha I(x, t)(x, t)+d_{4} I_{t t}
\end{aligned}
$$

$$
\begin{aligned}
\mathscr{L}_{R}=\frac{R(x, t+\ell)-R(x, t)}{\ell} & \\
& -(-r-\delta+\alpha I(x, t)) R(x, t)-\kappa E(x, t)-\gamma I(x, t) \\
& -d_{5}\left[\frac{R(x-h, t+\ell)-2 R(x, t+\ell)+R(x+h, t+\ell)}{h^{2}}\right] \\
& -R_{t}+\kappa E(x, t)+\gamma I(x, t)-r R(x, t)-\delta R(x, t) \\
& +\alpha I(x, t) R(x, t)+d_{5} R_{x x}
\end{aligned}
$$

Expanding
$S(x \pm h, t), V(x \pm h, t), E(x \pm h, t), I(x \pm h, t), R(x \pm h, t)$

and

$S(x, t+\ell), V(x, t+\ell), E(x, t+\ell), I(x, t+\ell), R(x, t+\ell)$

in (16) as Taylor series about $(x, t)$ leads to

$$
\begin{gathered}
\mathscr{L}_{S}=-\frac{1}{12} d_{1} h^{2} \frac{\partial^{4} S}{\partial x^{4}}+l\left[\frac{1}{2} \frac{\partial^{2} S}{\partial t^{2}}-d_{1} \frac{\partial^{3} S}{\partial x^{2} \partial t}\right]+\ldots, \\
\mathscr{L}_{V}=-\frac{1}{12} d_{2} h^{2} \frac{\partial^{4} S}{\partial x^{4}}+l\left[\frac{1}{2} \frac{\partial^{2} S}{\partial t^{2}}-d_{2} \frac{\partial^{3} S}{\partial x^{2} \partial t}\right]+\ldots, \\
\mathscr{L}_{I}=-\frac{1}{12} d_{3} h^{2} \frac{\partial^{4} S}{\partial x^{4}}+l\left[\frac{1}{2} \frac{\partial^{2} S}{\partial t^{2}}-d_{3} \frac{\partial^{3} S}{\partial x^{2} \partial t}\right]+\ldots, \\
\mathscr{L}_{I}=-\frac{1}{12} d_{4} h^{2} \frac{\partial^{4} S}{\partial x^{4}}+l\left[\frac{1}{2} \frac{\partial^{2} S}{\partial t^{2}}-d_{4} \frac{\partial^{3} S}{\partial x^{2} \partial t}\right]+\ldots \\
\mathscr{L}_{R}=-\frac{1}{12} d_{5} h^{2} \frac{\partial^{4} S}{\partial x^{4}}+l\left[\frac{1}{2} \frac{\partial^{2} S}{\partial t^{2}}-d_{5} \frac{\partial^{3} S}{\partial x^{2} \partial t}\right]+\ldots,
\end{gathered}
$$

Equation(21) verify that the method are (11)-(15)

$$
O\left(h^{2}+\ell\right) \text { as } h, \ell \rightarrow 0,
$$

\section{Implementations}

The derivative $\frac{\partial S}{\partial x}$ in the boundary conditions (3), is approximated by the second-order, central-difference replacement

$$
\frac{\partial S}{\partial x}=\frac{S(x+h, t)-S(x-h, t)}{2 h}+O\left(h^{2}\right)
$$

with a similar replacement being made to

$$
\frac{\partial V}{\partial x}, \frac{\partial E}{\partial x}, \frac{\partial I}{\partial x} \text { and } \frac{\partial R}{\partial x}
$$

The implementation of the boundary condition, the method (11)-(15) yield on $x=-L$ and $x=L$,

$$
\begin{gathered}
\tilde{S}_{1}^{n}=\tilde{S}_{-1}^{n} \quad \text { and } \quad \tilde{S}_{M+1}^{n}=\tilde{S}_{M-1}^{n} \\
\tilde{V}_{1}^{n}=\tilde{V}_{-1}^{n} \quad \text { and } \quad \tilde{V}_{M+1}^{n}=\tilde{V}_{M-1}^{n} \\
\tilde{E}_{1}^{n}=\tilde{E}_{-1}^{n} \text { and } \quad \tilde{E}_{M+1}^{n}=\tilde{E}_{M-1}^{n} \\
\tilde{I}_{1}^{n}=\tilde{I}_{-1}^{n} \quad \text { and } \quad \tilde{I}_{M+1}^{n}=\tilde{I}_{M-1}^{n} \\
\tilde{R}_{1}^{n}=\tilde{R}_{-1}^{n} \quad \text { and } \quad \tilde{R}_{M+1}^{n}=\tilde{R}_{M-1}^{n}
\end{gathered}
$$

Let

$$
\begin{gathered}
\tilde{\mathbf{S}}^{n+1}=\left[\tilde{S}_{0}^{n+1}, \tilde{S}_{1}^{n+1}, \ldots, \tilde{S}_{M}^{n+1}\right]^{T}, \tilde{\mathbf{v}}^{n+1}=\left[\tilde{V}_{0}^{n+1}, \tilde{V}_{1}^{n+1}, \ldots, \tilde{V}_{M}^{n+1}\right]^{T}, \\
\tilde{\mathbf{E}}^{n+1}=\left[\tilde{E}_{0}^{n+1}, \tilde{E}_{1}^{n+1}, \ldots, \tilde{E}_{M}^{n+1}\right]^{T}, \tilde{\mathbf{I}}^{n+1}=\left[\tilde{I}_{0}^{n+1}, \tilde{I}_{1}^{n+1}, \ldots, \tilde{I}_{M}^{n+1}\right]^{T},
\end{gathered}
$$




$$
\tilde{\mathbf{R}}^{n+1}=\left[\tilde{R}_{0}^{n+1}, \tilde{R}_{1}^{n+1}, \ldots, \tilde{R}_{M}^{n+1}\right]^{T}
$$

where $T$ denotes transpose. The modification to the formulae of the families of method (11)-(15), and their implication, are as follows.

Taking $m=0$, M in (7)-(11) using (26)-(30) gives $m=0$,

$$
\begin{aligned}
& \left(1+2 d_{1} p\right) \tilde{S}_{0}^{n+1}-2 d_{1} p \tilde{S}_{1}^{n+1} \\
= & {\left[1-\left(\beta_{1} \tilde{E}_{0}^{n}+\beta_{2} \tilde{I}_{0}^{n}+\alpha \tilde{I}_{0}^{n}-\phi-r\right) \ell\right] \tilde{S}_{0}^{n} } \\
& +r \ell+\delta \ell \tilde{R}_{0}^{n}+\theta \ell \tilde{V}_{0}^{n} \\
& \left(1+2 d_{2} p\right) \tilde{V}_{0}^{n+1}-2 d_{2} p \tilde{V}_{1}^{n+1} \\
= & {\left[1+\left(-\beta_{2} \beta \tilde{E}_{0}^{n}-\beta_{3} \tilde{I}_{0}^{n}+\alpha \tilde{I}_{0}^{n}-r-\theta\right) \ell\right] \tilde{V}_{0}^{n} } \\
+ & \phi \ell \tilde{S}_{0}^{n} \\
& \left(1+2 d_{3} p\right) \tilde{E}_{0}^{n+1}-2 d_{3} p \tilde{E}_{1}^{n+1} \\
& =\left[1+\left(\beta_{1} \tilde{S}_{0}^{n}+\beta_{3} \tilde{V}_{0}^{n}+\alpha \tilde{I}_{0}^{n}-w_{1}\right) \ell\right] \tilde{E}_{0}^{n} \\
& +\beta_{2} \ell \tilde{I}_{0}^{n} \tilde{S}_{0}^{n}+\beta_{4} \ell \tilde{I}_{0}^{n} \tilde{V}_{0}^{n} \\
& \left(1+2 d_{4} p\right) \tilde{I}_{0}^{n+1}-2 d_{4} p \tilde{I}_{1}^{n+1} \\
= & {\left[1+\left(-w_{2}+\alpha \tilde{I}_{0}^{n}\right) \ell\right] \tilde{I}_{0}^{n}+\sigma \ell \tilde{E}_{0}^{n} } \\
& \left(1+2 d_{5} p\right) \tilde{R}_{0}^{n+1}-2 d_{5} p \tilde{R}_{1}^{n+1} \\
= & {\left[1+\left(-r-\delta+\alpha \tilde{I}_{0}^{n}\right) \ell\right] \tilde{R}_{0}^{n}+\kappa \ell \tilde{E}_{0}^{n} } \\
& +\gamma \ell \tilde{I}_{0}^{n}
\end{aligned}
$$

$m=M$,

$$
\begin{aligned}
& -2 d_{1} p \tilde{S}_{M-1}^{n+1}+\left(1+2 d_{1} p\right) \tilde{S}_{M}^{n+1} \\
= & {\left[1-\left(\beta_{1} \tilde{E}_{M}^{n}+\beta_{2} \tilde{I}_{M}^{n}+\alpha \tilde{I}_{M}^{n}-\phi-r\right) \ell\right] \tilde{S}_{M}^{n} } \\
& +r \ell+\delta \ell \tilde{R}_{M}^{n}+\theta \ell \tilde{V}_{M}^{n} \\
- & 2 d_{2} p \tilde{V}_{M-1}^{n+1}+\left(1+2 d_{2} p\right) \tilde{V}_{M}^{n+1} \\
= & {\left[1+\left(-\beta_{2} \beta \tilde{E}_{M}^{n}-\beta_{3} \tilde{I}_{M}^{n}+\alpha \tilde{I}_{M}^{n}-r-\theta\right) \ell\right] \tilde{V}_{M}^{n} } \\
+ & \phi \ell \tilde{S}_{M}^{n} \\
- & 2 d_{3} p \tilde{E}_{M-1}^{n+1}+\left(1+2 d_{3} p\right) \tilde{E}_{M}^{n+1} \\
= & {\left[1+\left(\beta_{1} \tilde{S}_{M}^{n}+\beta_{3} \tilde{V}_{M}^{n}+\alpha \tilde{I}_{M}^{n}-w_{1}\right) \ell\right] \tilde{E}_{M}^{n} } \\
+ & \beta_{2} \ell \tilde{I}_{M}^{n} \tilde{S}_{M}^{n}+\beta_{4} \ell \tilde{I}_{M}^{n} \tilde{V}_{M}^{n} \\
& -2 d_{4} p \tilde{I}_{M-1}^{n+1}+\left(1+2 d_{4} p\right) \tilde{I}_{M}^{n+1} \\
& =\left[1+\left(-w_{2}+\alpha \tilde{I}_{M}^{n}\right) \ell\right] \tilde{I}_{M}^{n}+\sigma \ell \tilde{E}_{M}^{n}
\end{aligned}
$$


solve PDEs will be reduced significant.

\section{E. Numerical Expriments}

To test an implicit method (7) the model (1) is solved and the initial/boundary-value consisting of the Eqs. (2) and (3) by using the set of parameters:

$$
\begin{aligned}
& \beta=0.514, \beta_{E}=0.25, \beta_{I}=1, \mu=5.5 \times 10^{-8}, r=7.14 \times 10^{-5} \\
& \kappa=1.857 \times 10^{-4}, \beta_{v}=0.1, \alpha=9.3 \times 10^{-6}, \sigma=\frac{1}{2}, \theta=\frac{1}{365} \\
& \gamma=\frac{1}{5}, \delta=\frac{1}{365},
\end{aligned}
$$

and the diffusion rate, $d_{i}=0.005,(i=1,2, \ldots, 5)$.

In this experiment, taking $h=0.2$ so that $M=20$, giving the discretization $x_{i}(i=0,1, \ldots, 20)$ of the interval $-2 \leq x \leq 2$,

The initial conditions in (2) are distributed as follows (see Fig. 1- Fig. 3)

Experiment the initial conditions (i):

$$
\begin{array}{ll}
S_{0}(x)=0.86 \exp \left(-(x / 1.4)^{2}\right), & -2 \leq \mathrm{x} \leq 2 . \\
V_{0}(x)=0.1 \exp \left(-(x / 1.4)^{2}\right), & -2 \leq \mathrm{x} \leq 2 . \\
E_{0}(x)=0, & -2 \leq \mathrm{x} \leq 2 . \\
I_{0}(x)=0.04 \exp \left(-x^{2}\right), & -2 \leq \mathrm{x} \leq 2 . \\
R_{0}(x)=0, & -2 \leq \mathrm{x} \leq 2 .
\end{array}
$$

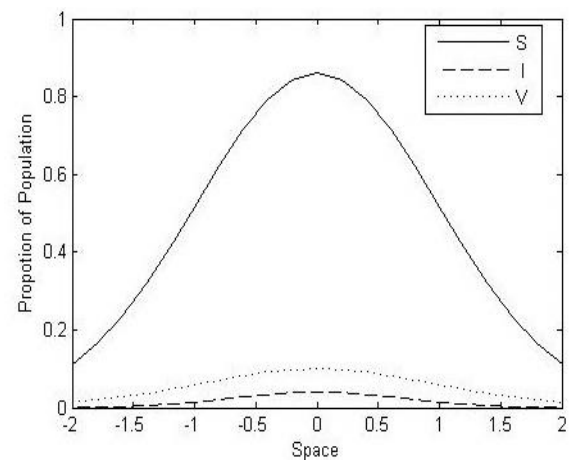

Fig. 1. Solution with initial condition (i).
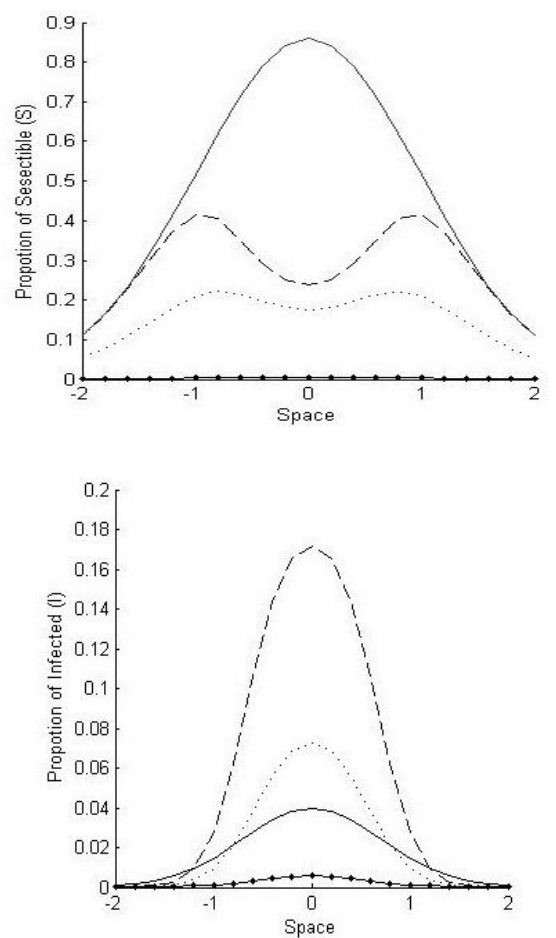
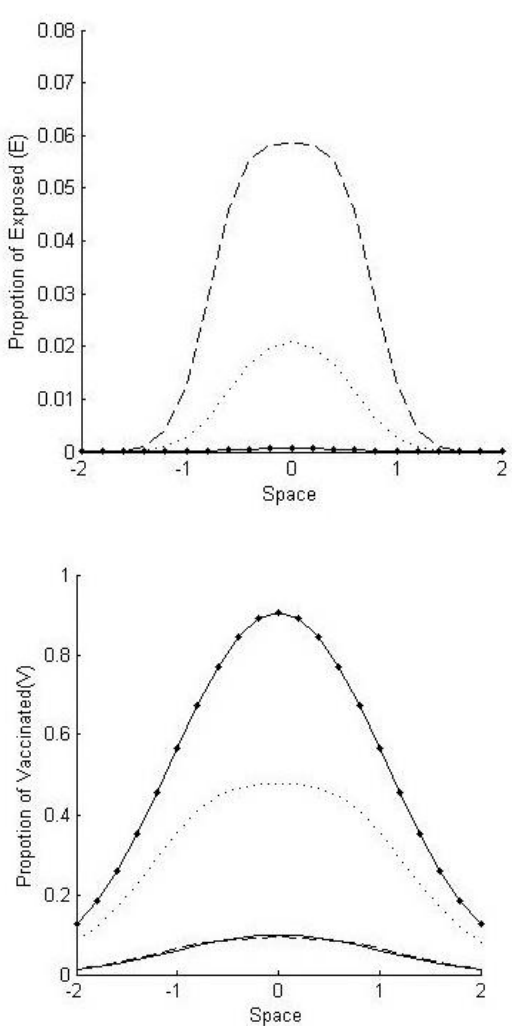

Fig. 2. Solution with initial condition (i) and with diffusion for cases $\phi=0.001(---), \phi=0.05(\ldots)$ and $\phi=0.5(\longrightarrow)$ at $t=20$ days and $t=0$ day

Fig. 2 shows the output with initial condition at $t=0$ and $\mathrm{t}$ $=20$ days the susceptible population is spreads all over the domain $[-2,2]$. At $t=20$ days case $\phi=0,001$ the infection population is spreads all over the domain $[-2,2]$. Case $\phi=0.05$ the infection population spreads in domain $[-1.8,1.8]$ and case $\phi=0.5$ the infection population is spreads in domain $[-1.6,1.6]$. The exposed case $\phi=0.001, \phi=0.05$ and $\phi=0.5$ is spreads in domain $[-1.8,1.8],[-1.6,1.6]$ and $[-1.2,1.2]$ respectively. The increment of vaccinated population enlarges the number rate of vaccinated.

Experiment the initial conditions (ii):

$$
\begin{aligned}
& S_{0}=0.86 \operatorname{Sech}^{2}(10 x), \quad-2 \leq x \leq 2 . \\
& V_{0}=0.10 \operatorname{Sech}^{2}(10 x), \quad-2 \leq x \leq 2 . \\
& E_{0}=0, \quad-2 \leq x \leq 2 . \\
& I_{0}=\left\{\begin{array}{cc}
0, & -2 \leq x<-0.5, \\
0.04, & -0.5 \leq x \leq 0.5, \\
0, & 0.5<x \leq 2 .
\end{array}\right. \\
& R_{0}=0, \quad-2 \leq x \leq 2 .
\end{aligned}
$$

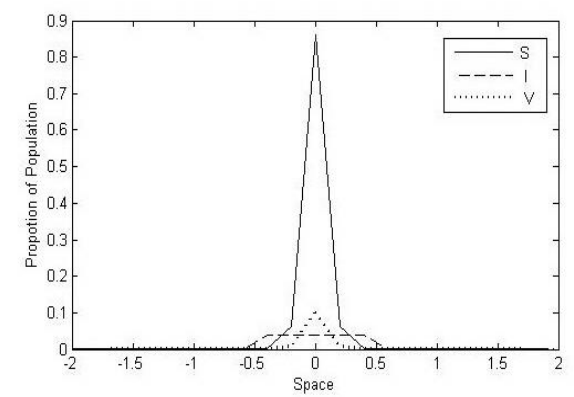

Fig. 3. Solution with initial condition (ii). 

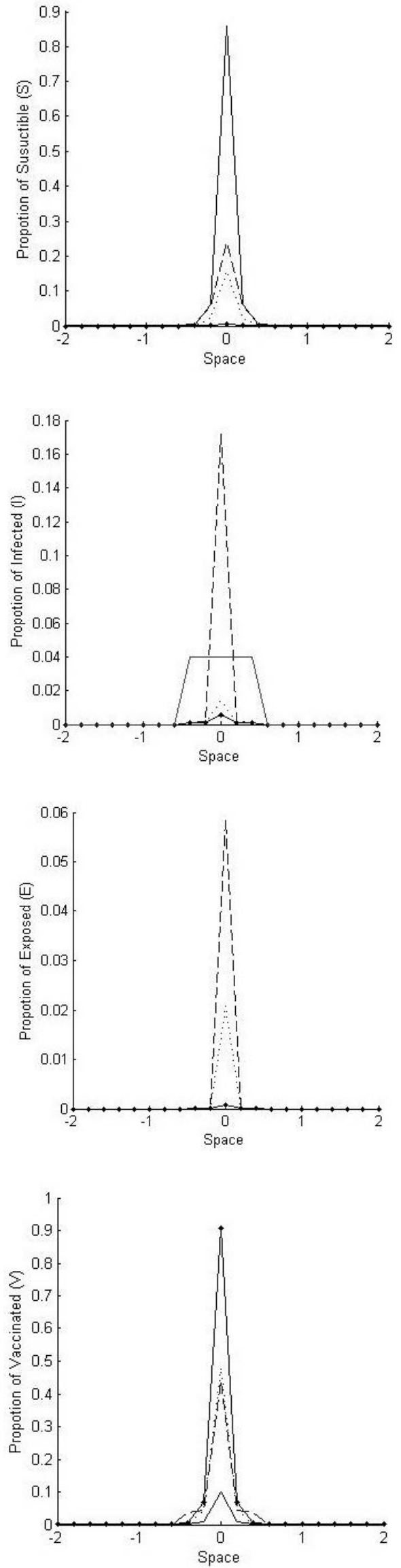

Fig. 4. Solution with initial condition (ii) and with diffusion for cases $\phi=0.001(---), \phi=0.05(\ldots)$ and $\phi=0.5(\longrightarrow)$ at $t=20$ days and $t=0$ day

Fig. 4 shows the output with initial condition (ii). The susceptible population is steadily spreading to domain $[-0.6$,
0.6]. After $t=20$ days it spreads to the whole domain [-2, 2] as $\phi=0.001, \phi=0.05$ and $\phi=0.5$ respectively. Infection is spreading to domain $[-0.6,0.6]$ at $t=0$ and $t=20$ days. At $t=$ 20 days recovery is taking place in the domain $[-0.6,0.6]$ as case $\phi=0.001$ and $\phi=0.05$ it has spread to domain $[-0.4$, $0.4]$ for case $\phi=0.5$. The increment of vaccinated population enlarges the number rate of vaccinated

\section{CONCLUSION}

An implicit finite-difference scheme has been developed and implemented in this paper for computing the solutions of the SVEIR model with vaccination in one dimension(1). The accuracy of the constructed method is first order in time and second-order in space. The numerical experiments showed that the numerical solution obtained from the constructed method is coincided with the solution in [2]. Moreover, the numerical results showed that the dynamic behavior of influenza depends on the initial distributions and the diffusion rate.

\section{ACKNOWLEDGMENT}

The work was supported by the National Centre of Exellence in Mathematics under the Commission on Higher Educational, Ministry of Education, Thailand.

\section{REFERENCES}

[1] K. Dietz and D. Schenzle, "Mathematcal models for infectious disease statistics," in A. C. Atkinson, S. E. Fienberg(Eds.), Centeary Volume of the International Statistical Institute, Berlin: Springer-Verlag, 1985

[2] D. J. D. Earn, J. Dushoff, and S. A. Levin, "Ecolgy and evolution of the flu," Trend Ecol., vol. 17, pp. 334-340, 2002.

[3] J. Lin, V. Andreasen, and S. A. Levin, "Dynamics of influenza A drift: the linear three-strain model," J. Epidemiol. Math. Biosci., vol. 162, pp 33-51, 1999.

[4] R. J. Webby, "Webster, Are we ready for pandemic influenza?" Science, vol. 302, pp 1519-1522, 2003.

[5] M. Samsuzzoha, M. Singh, and, D. Lucy, "A numerical study on an influenza epidemic model with vaccination and diffusion," Applied Mathematics and Computtation, vol. 219, pp. 122-141.

[6] K. Dietz and D. Schenzle, "Mathematcal models for infectious disease statistics," in A. C. Atkinson, S. E. Fienberg(Eds.), Centeary Volume of the International Statistical Institute, Berlin: Springer-Verlag, pp. 12, 1985.

[7] R. M. Anderson and R. M. May, "Age-related changes in the rate of disease transmission: implication for the design of vaccination programmes," Hyg. Camb., vol. 94, 1985, pp. 365-436.

[8] I. M. Longini, M. E. Ackerman, A. Nizam, and Y. Yang, "Containing pandemic influenza with antiviral agents," Am. J. Epidemiol., vol. 159, pp. 623-633. 2004

[9] C. Viboud, P. Y. Boelle, F. Carrat, A. J. Valleron, and A. F. Prahault, "Prediction of the spread of influenza epidemics by the method of analogues," Am. J. Epiemiol., vol. 158, pp. 996-1006, 2003.

[10] C. Viboud, P. Y. Boelle, K. Pakdaman, F. Carrat, A. J. Valleron, and A Flahault, "Influenza epidemics in the United States, France, and Australia," Emerg. Infect. Dis., vol. 10, pp. 32-39, 2004.

[11] R. M. Anderson and R. M. May, "Vaccination against rubella and measles; quantitative investigations of different policies," Hyg. Camb., vol. 90, 1983, pp. 259-325.

[12] R. M. Anderson and R. M. May, "Age-related changes in the rate of disease transmission: implication for the design of vaccination programmes," Hyg. Camb., vol. 94, 1985, pp. 365-436.

[13] D. Greenhalgh and R. Das, "An SIR epidemic model with a contact rate depending on population density," in O. Arino, O. Axelrod, M. Langlais(Eds.), Mathematcal Population Dynamics: Analysis of Heterogeneity, Winnipeg: Wuerz Publishing, 1995.

[14] M. Samsuzzoha, M. Singh, and D. Lucy, "Uncertainty and sensitivity analysis of the basic reproduction number of a vaccinated epidemic 
model of influenza," Applied Mathematical Modelling, vol. 37, pp. 903-915.

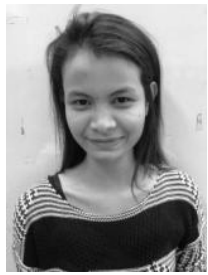

Dussadee Somjaivang was born on $30^{\text {th }}$ September 1989. She was graduated bachelor degree of science (Applied Mathematics) at King Mongkut's University of Technology Thonburi, 2011.

Now, she is studying master degree of science (Applied mathematics) in department of Mathematics at King Mongkut's University of Technology Thonburi.

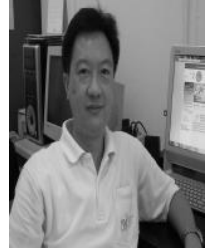

Settapat. Chinviriyasit is currently employed as an associated professor and a doctor in mathematics at King Mongkut's University of Technology Thonburi, Thailand.

He obtained his PhD from Brunel university (UK). His interested research is as follow: numerical modeling of differential equations by using finite difference and finite element methods, mathematical modeling in ecology and biology, applied dynamical systems, Computational mathematics. 